\title{
ESTADO ACTUAL DE LA LUCHA CONTRA EL CANCER DEL UTERO EN CUBA
}

\author{
Prof. Eduardo Cutié León* \\ Prof. Pedro Valdés Vivó** \\ Prof. Armando Peralta Quintero***
}

\section{Antecedentes}

El control en escala nacional del cáncer uterino, constituye una meta del Ministerio de Salud Pública. Siguiendo las orientaciones de los organismos internacionales, -Unión Internacional contra el Cáncer y la Organización Mundial de la Saludy las experiencias de algunos países, tanto occidentales como socialistas, Cuba se propone obtener el mejor resultado de la detección preclínica del cáncer del útero, por medio del citodiagnóstico. Los estudios preliminares del Programa de Detección Preclínica del Cáncer Uterino, se llevaron a cabo durante los últimos nueve meses de 1966, a través de una unidad piloto, anexa al Instituto de Oncología y Radiobiología de La Habana. Durante los meses siguientes la detección fue extendiéndose desde el área metropolitana de La Habana a todas las provincias de la Nación.

Un aspecto importante de esta etapa preliminar lo fue el establecimiento de un procedimiento ejecutivo para el registro primario de las actividades, su acopio sistemático y su ulterior análisis estadístico.

Por último, desde mediados de 1967, se han estado estudiando los planes perspectivos que permitan lle- gar dentro de diez años, a la meta de la detección anual de un millón de mujeres, o sea prácticamente la mitad de la población femenina del País, lo que permitirá el re-examen de cada mujer, con intervalos de tres años.

La experiencia hasta ahora recogida, parece demostrar que la meta del control del cáncer uterino, será factible, pues la actual estructura organizativa de la medicina cubana, permite, que se allanen obstáculos que en la situación pre-revolucionaria, hubieran sido insuperables.

En la actualidad el Programa de Detección Preclínica del Cáncer Uterino se encuentra bajo la dirección de una Comisión Nacional, adscrita al Vice-Ministerio de Asistencia y Docencia Médica del Ministerio de Salud Pública, constituída por oncólogos, gineco-obstetras, patólogos, estadísticos y responsables de organizaciones de masas.

* Departamento de Obstetricia y Ginecología de la Escuela de Medicina de la Universidad de La Habana. Secretario de la Sociedad Cubana de Obstetricia y Ginecología.

** Departamento de Obstetricia y Ginecología de la Escuela de Medicina de la Universidad de La Habana.

*** Departamento de Obstetricia y Ginecología de la Escuela de Medicina de la Universidad de La Habana. 
La coordinación del trabajo en las provincias se efectúa a través de las ocho Comisiones Provinciales del Programa, las que tienen una composición análoga a la Comisión Nacional. Estas comisiones provinciales a su vez coordinarán el desenvolvimiento del Programa en sus respectivas regionales y a través de éstas en las diferentes unidades ejecutoras de Salud Pública, (Hospital provincial, hospitales regionales, laboratorios regionales, policlínicos).

\section{Material y Método}

\section{Captación del Paciente:}

El Programa alcanza a todas las mujeres embarazadas, a las cuales se les toma la muestra citológica como examen obligado durante su atención pre-natal; a toda paciente que acude a una consulta ginecológica, independientemente de su motivo de consulta, estado civil o edad y por último incluye a la mujer sana soltera de más de 30 años de edad o casada de cualquier edad que es captada por medio de campañas de divulgación, utilizando ampliamente los recursos de la prensa, la radio y la televisión, así como la cooperación valiosísima de la Organización Nacional de los Comités de Defensa de la Revolución que lleva a cabo un trabajo sistemático de movilización por medio de reuniones de divulgación, audiencias sanitarias y visitas domiciliarias.

\section{Toma de la Muestra:}

Es realizada en los policlínicos integrales y hospitales regionales por personal médico o auxiliar entrenado para este fin, de acuerdo con las técnicas internacionalmente aceptadas (raspado superficial o aspiración con pipeta).

\section{Tamizaje Primario:}

Se efectúa en el Laboratorio Regional con procedimientos de fijación, coloración y observación estandarizadas, por citotécnicas especializadas en cursos de 12 meses de duración y bajo el control y supervisión de médicos citólogos $\mathrm{y} / \mathrm{o}$ anatomopatólogos.

En adelante la conducta a seguir depende del resultado de la prueba citológica que podrá ser:

a) No útil para diagnóstico: por defectos o fallas técnicas que la inutilicen para su estudio microscópico.

b) Negativo.

C) Sospechoso.

d) Positivo.

$\mathrm{Si}$ el resultado fuera negativo, se confecciona el carné que se hace llegar a la interesada y el informe del citodiagnóstico es archivado en el policlínico integral correspondiente en un tarjetero calendario para su nueva realización a los tres años y así sucesivamente, durante el resto de la vida de la mujer.

\section{Tamizaje Secundario:}

Si el resultado fuera no útil, se cita prontamente a la paciente, valiéndose para su localización de la labor de enfermería de terreno de que disponen todos nuestros policlínicos, pero si el resultado fuera sospechoso o positivo, se localiza rápidamente a la paciente por los mecanismos ya antes mencionados, más la intervención directa del Laboratorio Provincial o Regional que en esta situación puede disponer del personal del hospital destinado a esta tarea y remitirla a la Consulta Especial de Patología del Cuello Uterino que es atendida por un médico gine-obste- 
tra y un médico citólogo, donde es adecuada y exhaustivamente estudiada, hasta que se establece un diagnóstico definitivo; en estas consultas especiales se realiza el re-examen citológico de confirmación con el complemento del estudio colposcópico y biopsia dirigida, contando además con los recursos necesarios de camas y salones quirúrgicos para el estudio biópsico definitivo que es siempre la biopsia por conización fría del cuello uterino o biopsia dirigida cuando la lesión es macroscópicamente evidente.

\section{Tratamiento:}

En cuanto a su tratamiento es suficiente consignar que se conduce de acuerdo con las normas reconocidas en este aspecto y publicadas en el $\mathrm{Li}$ bro de Normas Ginecológicas que recopila las conclusiones de la I Jornada de Normación de la Ginecología celebrada en el año 1967 el cual incluye no solo el tratamiento de las pacientes confirmadas de cáncer, sino también el de las lesiones precancerosas e inflamatorias del cuello uterino.

\section{Control y Seguimiento:}

Todas las pacientes con diagnóstico confirmado de cáncer son tratadas y controladas según normas establecidas por un período de cinco años, así como durante no menos de doce meses todos los casos sospechosos que se negativizan.

\section{Control Estadístico:}

Como es fácil comprender sólo procediendo sistemáticamente, de acuerdo con un plan perfectamente definido podrá llegarse a detectar entre una gran masa de mujeres, aquellas ya afectadas preclínicamente o clínicamente por el cáncer uterino y poder someterlas en tiempo oportuno a un tratamiento útil y efectivo y esto sólo es posible mediante un estricto control estadístico que permita que la labor realizada sea convenientemente registrada para que sea utilizable para su estudio epidemiológico.

Todos los resultados negativos son archivados en un tarjetero calendario en el policlínico integral para su repetición a los tres años y todos los resultados sospechosos y positivos se segregan a un archivo especial adicionándoles los datos de la confirmación del diagnóstico, tratamiento definitivo y seguimiento, siendo este control doble pues se realiza tanto en el laboratorio como en el policlínico integral correspondiente para de esta forma lograr un control seguro de las pacientes.

Todos los laboratorios, mensualmente, informan su trabajo a su correspondiente Departamento de Estadística Provincial del Ministerio de Salud Pública y a la Comisión Provincial del Programa y éstas a su vez a sus homólogas nacionales: Departamento Nacional de Estadísticas del Ministerio de Salud Pública y Comisión Nacional del Programa.

\section{Cáncer Endometrial:}

Desde hace ya varios años es norma la utilización del legrado uterino diagnóstico en toda paciente con sangramiento uterino postmenopáusico o anormal, siguiéndose los procedimientos de diagnóstico y tratamiento establecidos en la I Jornada de Normación de la Ginecología, hecho éste que justifica el incremento tan notable de los legrados diagnósticos registrados en los dos últimos años. 


\section{Coriocarcinoma :}

En nuestro País desde finales de 1968 existe el Registro Central de Enfermedad Trofoblástica y se encuentra establecido el procedimiento de control y seguimiento de las pacientes de enfermedad trofoblástica benigna o maligna, contándose con los recursos necesarios (personal médico, camas, quimioterápicos, etc.) para su tratamiento de acuerdo a los últimos conocimientos que de esta afección se tienen.

\section{ANEXO}

COMIISION NACIONAL PARA LA DETECCION PRECLINICA DEL CANCER UTERINO

\section{Presidente,}

Director del Instituto de Oncología y Radiobiología de La Habana - Profesor Zoilo Marinello Vidaurreta.

\section{Secretario,}

Miembro del Grupo Nacional de Gineco-Obstetricia - Profesor Eduardo Cutié León.

\section{Secretaria,}

Instituto de Oncología y Radiobiología de La Habana - Dra. María Luisa Buch.

\section{Otros Miembros}

Jefe del Grupo Nacional de Gineco-Obstetricia Profesor Celestino Alvarez Lajonchere.
Jefe del Grupo Nacional de Anatomía Patológica - Profesor Israel Borrajero.

Miembro del Grupo Nacional de Gineco-Obstetricia - Profesor Ubaldo Farnot Cardoso.

Miembro del Grupo Nacional de Anatomía Patológica - Profesora Dora Gallego Pimentel. Miembro del Grupo Nacional de Gineco-Obstetricia - Dr. José Pagola Jiménez.

Jefe del Grupo Nacional de Estadísticas Médicas - Profesor Francisco Rojas Ochoa.

Responsable Nacional de Salud de los Comités de Defensa de la Revolución - Sr. Pedro Quirós.

PRUEBAS CITOLOGICAS

$1967-1969$

\begin{tabular}{lrrr}
\hline Años & Meta & Real & $\%$ \\
\hline 1967 & 75.000 & 76.254 & 101,6 \\
1968 & 239.000 & 205.722 & 86,0 \\
1969 & 350.000 & 308.658 & 88,1 \\
\hline
\end{tabular}

RECURSOS DEL PROGRAMA

1967-1969

\begin{tabular}{ccc}
\hline Años & $\begin{array}{l}\text { Laboratorios } \\
\text { funcionando }\end{array}$ & $\begin{array}{c}\text { Citotécnicas } \\
\text { graduadas }\end{array}$ \\
\hline 1967 & 7 & 12 \\
1968 & 13 & 47 \\
1969 & 17 & 46 \\
\hline
\end{tabular}


Vol. $\mathrm{XXII}$

\section{PRUEBAS CITOLOGICAS REALIZADAS POR UNIDADES} PROVINCIA HABANA METROPOLITANA

AÑo 1968

Hospitales

Policlínicos

Hosp. Mat. Mariano

\begin{tabular}{|c|c|}
\hline Womancoud & $\begin{array}{c}1 \times 1.000 \\
(23552)\end{array}$ \\
\hline
\end{tabular}

Hosp. Mat. América Arias

\begin{tabular}{|l|}
\hline \\
\hline \\
$10 \times 1.000)$ \\
$(6051)$
\end{tabular}

Hosp. Enrique Cabrera

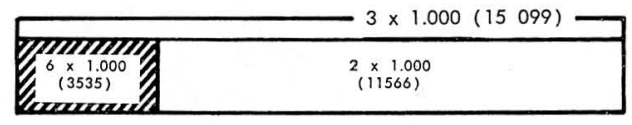

Mat. Inf. 10 de octubre

Hosp. Clínico Quirúrgico 10 de octubre

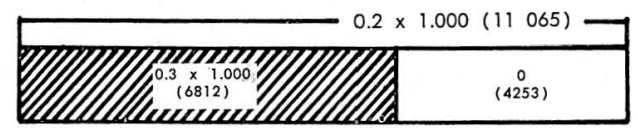

Hosp. Oncológico

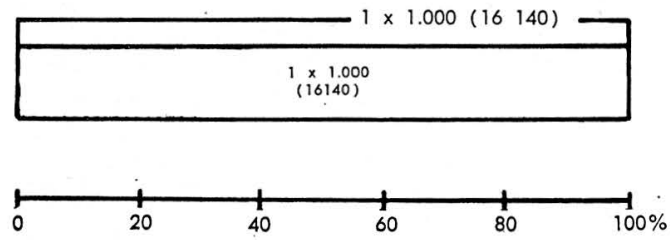

GRAFICO NN 1 
PRUEBAS CITOLOGICAS REALIZADAS POR UNIDADES PROVINCIA HABANA METROPOLITANA

ENERO- OCTUBRE DE 1969

Hospitales

Policlínicos

Hosp. Mat. Mariano

\begin{tabular}{|l|}
\hline$m+2 \times 1.000(18830)$ \\
$7 \times 1.000$ \\
$(4005)$
\end{tabular}

$4 \times 1.000(17607)$

Hosp. Mat. América Arias

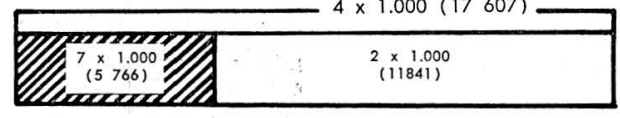

Hosp. Enrique Cabrera

\begin{tabular}{|c|c|}
\hline$=0$ & $2 \underset{(5858)}{x}$ \\
\hline
\end{tabular}

Mat. Inf. 10 de octubre

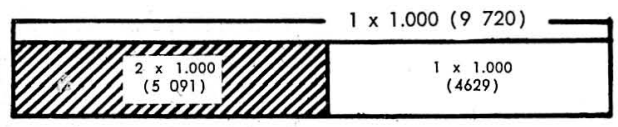

Hosp. Clínico Quirúrgico 10 de octubre

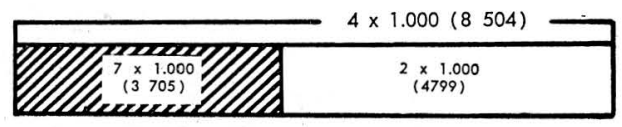

Hosp. Oncológico
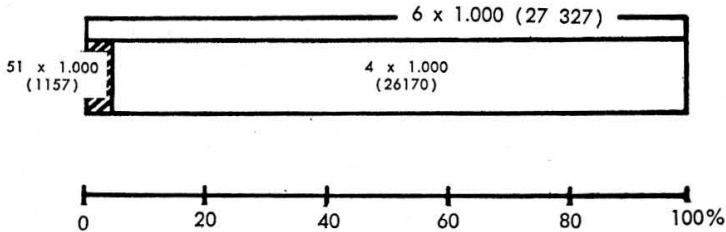

GRAFICO NN 2 